\title{
LA-8808-MS
}
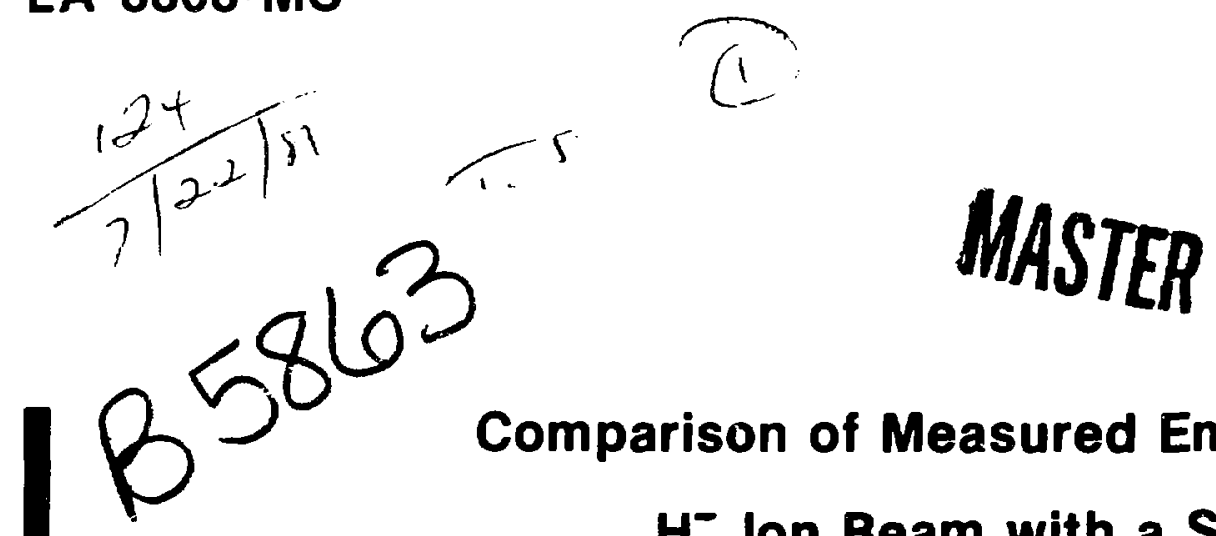

$\mathrm{H}^{-}$Ion Beam with a Simple Theory

$\frac{\pi}{5}$
$\frac{2}{0}$
$\frac{0}{0}$
$\frac{0}{0}$
$\frac{\pi}{0}$
$\frac{2}{2}$
$\frac{2}{2}$ 


\section{COMPARISON OF MEASURED EMITTANCE OF AN H- ION BEAM WITH A SIMPLE THEORY}

by

Paul Allison, Joseph D. Sherman, and H. Vernon Smith, Jr.

\section{ABSTRACT}

Ion beam phase space is frequently modeled with a simple theory having a few parameters; for example, the Kapchinski $i-V$ ladimirski $i(K-V)$ equations. For a real beam the normalized phase-space density $\partial^{2} i / \partial \times \partial \dot{x}$ in one transverse plane $x$, averaged over tíe other plane $y$, is usually far from that of a K-V distribution. We develop a simple theory based on plasma ion temperature that under some conditions gives a good description of the measured emittance of our $\mathrm{H}^{-}$ion beam at abcut $15 \mathrm{keV}$.

\section{GENERAL CONSIDERATIONS ON EMITTANCE}

The emittance of an ion beam has as its origins

1. the temperature of the ions in the plasma and the dimensions of the emitter,

2. ion-optical aberrations in the extraction and transport elements,

3. nonlinear space-charge forces, and

4. time-dependent fluctuations in the ion current (or in plasmas affecting the beam), leading to a time-averaged increase in emittance.

In a typical emittance scanner ${ }^{1}$ the phase-space contours are mapped as a function of threshold $t$, where

$$
t=\left(\partial^{2} i / \partial x \partial \dot{x}\right) /\left(\partial^{2} i / \partial x \partial \dot{x}\right)_{\max }
$$

a veraged over $y, \dot{y}$ space (where $\dot{x}=d x / d t$ ). For a given threshold $t_{0}$, the 
fraction $F$ of current and the normalized emittance $\varepsilon$ are given by

$$
\begin{aligned}
& F\left(t_{0}\right)=\iint_{t_{0} \leq t \leq 1}\left(\partial^{2} i / \partial x \partial \dot{x}\right) d x d \dot{x} / \iint_{0 \leq t \leq 1}\left(\partial^{2} i / \partial x \partial \dot{x}\right) d x d \dot{x} \\
& E\left(t_{0}\right)=\frac{1}{\pi} \iint_{t_{0} \leq t \leq 1} d x d \dot{x} / c .
\end{aligned}
$$

As a simple model ("Maxwellian"), we assume that the ions have a Maxwellian distribution of energies and a uniform spatial density at the plasma surface, and that the other sources of emittance (items 2, 3 , and 4 above) are negligible. Then $F\left(t_{0}\right)$ and $\varepsilon\left(t_{0}\right)$ are invariant in transport, even in the presence of linear space-charge forces.

This is because the phase-space density measured along the particle trajectories is constant and because the phase-space area inside a density contour is also constant (Liouville's theorem, Ref. 2, pp. 175-6); thus the individual integrals in Eqs. (2) and (3) are invariant, although the orientation of the phase space depends on the details of the beam transport. The threshoid parameter $\mathrm{t}$ gives the density contours through Eq. (1). The conclusion is that the emittance as a function of the beam fraction is the same at the measuring plane as at the ion emission plare, where we will make the theoretical calculation.

The normalized rms emittance, $\varepsilon_{\text {rms, }}$ is frequently defined as

$$
\varepsilon_{\mathrm{rms}}=\sqrt{\left.\left\langle\mathrm{x}^{2}\right\rangle \dot{x}^{2}\right\rangle-\langle x \dot{x}\rangle^{2}} / c
$$

For a $K-V$ distribution, ${ }^{3}$ the area of the phase-space ellipse divided by $\pi$ is $4 E_{\text {rms }}$. For the distributions considered here, we take $\langle x \dot{x}\rangle=0$ and calculate $\varepsilon_{4 r m s}=4 \varepsilon_{r m s}$ in accordarice with the definition by Lapostolle."

$$
\varepsilon_{4 r m s}=4 \sqrt{\left.\left\langle x^{2}\right\rangle \dot{x}^{2}\right\rangle} / c
$$

For our distributions, $\varepsilon_{4 \mathrm{rms}}$ encloses about $90 \%$ of the beam. 


\section{I. SLIT BEAM}

Most of our emittance measurements have been made for an $\mathrm{H}^{-}$beam extracted from a slit. For the "Maxwellian" model the threshold $t$ averaged over $y, \dot{y}$ is

$$
t=\exp \left(-m \dot{x}^{2} / 2 k T\right)
$$

The phase-space region defined by $t_{0}$ is then

$$
\begin{aligned}
& |x| \leqslant R \\
& |\dot{x}| \leqslant \sqrt{(2 k T / m) \quad \ln \left(1 / t_{0}\right)},
\end{aligned}
$$

where $2 R$ is the slit width.

$$
\begin{aligned}
& \text { Evaluation of Eqs. (2) and (3) leads to } \\
& F=\operatorname{erf}\left(\pi \varepsilon / 4 R \sqrt{2 k T / m c^{2}}\right) \text {. }
\end{aligned}
$$

To calculate $\varepsilon_{4}$ rms, we first determine $\left\langle\dot{x}^{2}\right\rangle$

$$
\begin{aligned}
\left\langle\dot{x}^{2}\right\rangle & =\frac{\int_{-R}^{R} d x \int_{-\infty}^{\infty} \dot{x}^{2} \exp \left(-m \dot{x}^{2} / 2 k T\right) d \dot{x}}{\int_{-R}^{R} d x \int_{-\infty}^{\infty} \exp \left(-m \dot{x}^{2} / 2 k T\right) d \dot{x}} \\
& =k T / m .
\end{aligned}
$$

Similarly, we find $\left\langle x^{2}\right\rangle=R^{2} / 3$, so that

$$
\varepsilon_{4 \mathrm{rms}}=\frac{4}{\sqrt{3}} \mathrm{R} \sqrt{\mathrm{kT} / \mathrm{mc^{2 }}},
$$




$$
F=\operatorname{erf}\left(\pi \varepsilon / \sqrt{6} \varepsilon_{4 r m s}\right),
$$

and

$$
F\left(\varepsilon_{4 r m s}\right)=\operatorname{erf}(\pi / \sqrt{6})=93 \% \text {. }
$$

\section{CIRCULAR-APERTURE BEAM}

In this case we have for an ion emitter of radius $R$

$$
\partial^{4} i / \partial x \partial \dot{x} \partial y \partial \dot{y}=C \exp \left(-m\left[\dot{x}^{2}+\dot{y}^{2}\right] / 2 k T\right), \text { for } x^{2}+y^{2} \leqslant R^{2} .
$$

Integration over $y, \dot{y}$ gives

$$
t=\sqrt{1-x^{2} / R^{2}} \exp \left(-m \dot{x}^{2} / 2 k T\right) .
$$

The region defined by $t$, is now somewhat complicated, and it is necessary to evaluate $F\left(t_{0}\right)$ and $\varepsilon\left(t_{0}\right)$ numerically, but it is still possible to evaluate $\epsilon_{r m s}$ analytically. As before $\left\langle\dot{x}^{2}\right\rangle=k T / m$, and

$$
\begin{aligned}
& \left\langle x^{2}\right\rangle=\frac{\int_{-R}^{R} x^{2} \sqrt{1-x^{2} / R^{2}} d x}{\int_{-R}^{R} \sqrt{1-x^{2} / R^{2}} d x}, \\
& =R^{2} / 4 .
\end{aligned}
$$

so that

$$
\varepsilon_{4 \mathrm{rms}}=2 R \sqrt{\mathrm{kT} / \mathrm{mc} \mathrm{c}^{2}} \text {, }
$$

in agreement with Eq. (4.42) of Ref. 2. The dependence of $\varepsilon$ on $F$ is very close to that for the slit case, as can be seen from $F i g .1$, where $\varepsilon / \varepsilon_{4 \mathrm{rms}}$ is plotted versus $F$ for the slit and for the aperture beams. 


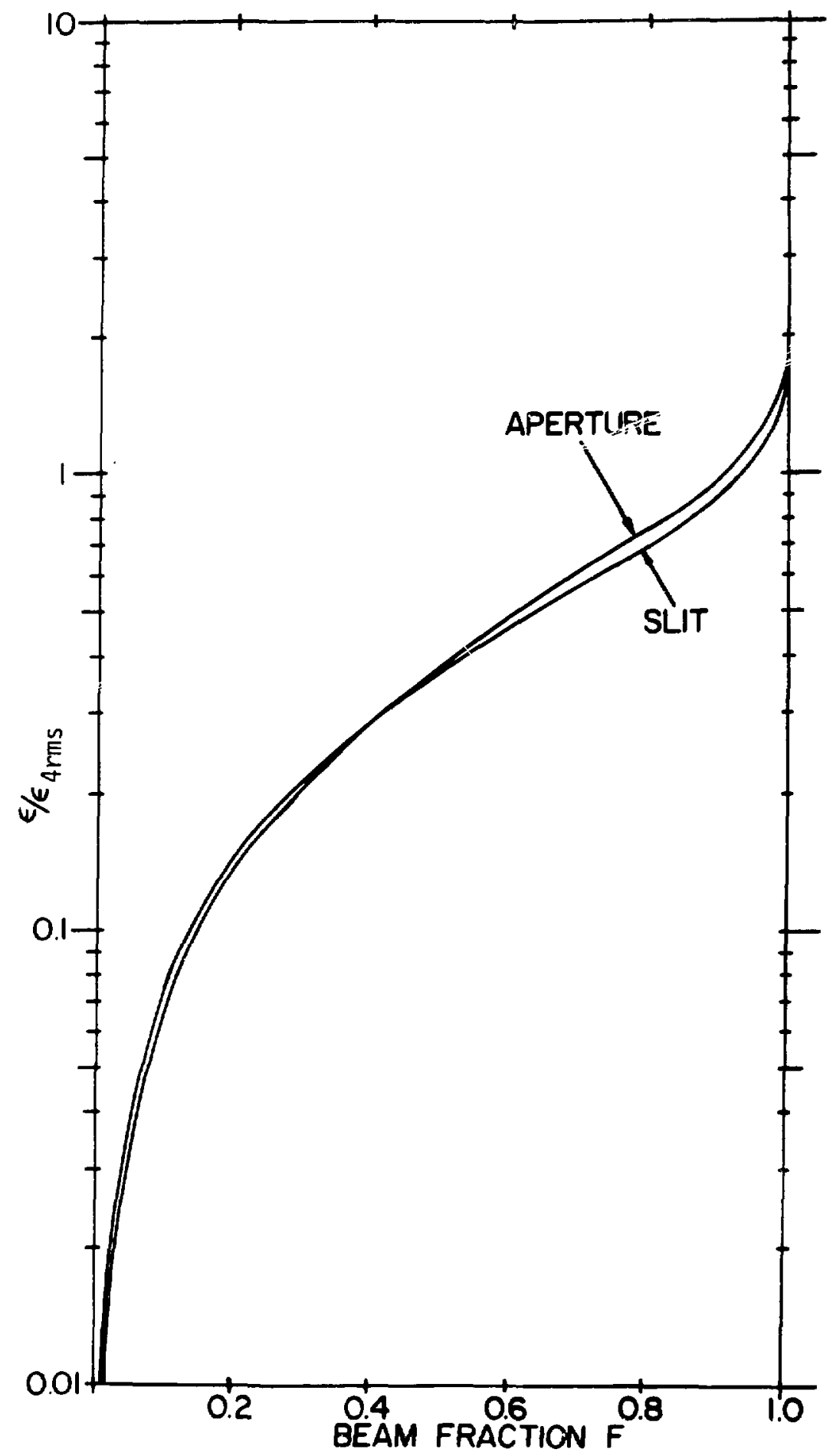

Fig. 1.

The quantity $\varepsilon / \varepsilon_{4 \text { rnis }}$ vs beam fraction $F$ for $s l i t$ and aperture ion emitters for the "Maxwellian" theory. 
For the slit case, $93 \%$ of the beam is contained within $\varepsilon_{4 \mathrm{rms}}$, compared with $89 \%$ for the aperture case. The 4 rms emittance of the slit beam is about $15 \%$ larger than that for the aperture beam, according to Eqs. (7) and (10).

IV. OTHER MODELS

A model ("Gaussian") that gives a better fit to our slit data assumes a Gaussian in both $x$ and $\dot{x}$. To make comparisons, we choose $\left\langle x^{2}\right\rangle=R^{2} / 3$. By this measure, the emission radii are the same for the "Gaussian" and the "Maxwellian" distributions. Then for the slit or aperture beams

$$
t=\exp \left(-3 x^{2} / 2 R^{2}-m \dot{x}^{2} / 2 k T\right), \quad \text { for }|x|<\infty,|\dot{x}|<\infty .
$$

Evaluation of Eqs. (2), (3), and (5) leads to

$$
\begin{aligned}
& F(\varepsilon)=1-\exp \left(-2 \varepsilon / \varepsilon_{4 r m s}\right), \\
& \varepsilon_{4 r m s}=\frac{4}{\sqrt{3}} R \sqrt{k T / m c^{2}}, \text { and } \\
& F\left(\varepsilon_{4 \text { rms }}\right)=86 \% .
\end{aligned}
$$

These results are very close to those for the "Maxwellian" model.

The frequently used $K-V$ distribution has a uniform density in $x, \dot{x}$ space (or any other two-dimensional projection). We choose $\left\langle\dot{x}^{2}\right\rangle=k T / m$, so that the distribution at a waist is uniform for

$$
x^{2} / R^{2}+\dot{x}^{2} /(4 k T / m) \leq 1
$$

Then $\left\langle x^{2}\right\rangle=R^{2} / 4$ so that

$$
\begin{aligned}
& \varepsilon_{4 \mathrm{rms}}=2 \mathrm{R} \sqrt{\mathrm{kT} / \mathrm{mc}^{2}} \text {, and } \\
& F=\varepsilon / \varepsilon_{4 \mathrm{rms}} .
\end{aligned}
$$


Emittance was measured with a scanner ${ }^{1}$ having two slits, between which is a pair of electrostatic deflecting plates to analyze the current distribution as a function of angle (see Fig. 2). These measurements are made as a function of position across the beam. The threshold $t$ is given by the current passing through the rear slit into the scanner Faraday cup divided by the maximum value. The measured dependence of emittance on beam fraction was obtained by computer integration of these data using the definitions of Eqs. (1) through (3).

The data ${ }^{5}$ for a $17-\mathrm{keV}, 80-\mathrm{mA} \mathrm{H}^{-}$beam extracted from a 10 - by $0.5-\mathrm{mm}^{2} \mathrm{slit}$ are shown in Fig. 3, and $k T$ was chosen to normalize the curve calculated from Eq. (6) to the data at $F=63 \%$. The $10-\mathrm{mm}$ dimension of the slit is in the bend $p l$ ane, $x$. The beam was transported from extraction through a $90^{\circ} \mathrm{n}=0.85$ magnet to the scariner $19 \mathrm{~cm}$ past the magnet. For the $x-p l$ ane data, $k T=5 \mathrm{ev}$,

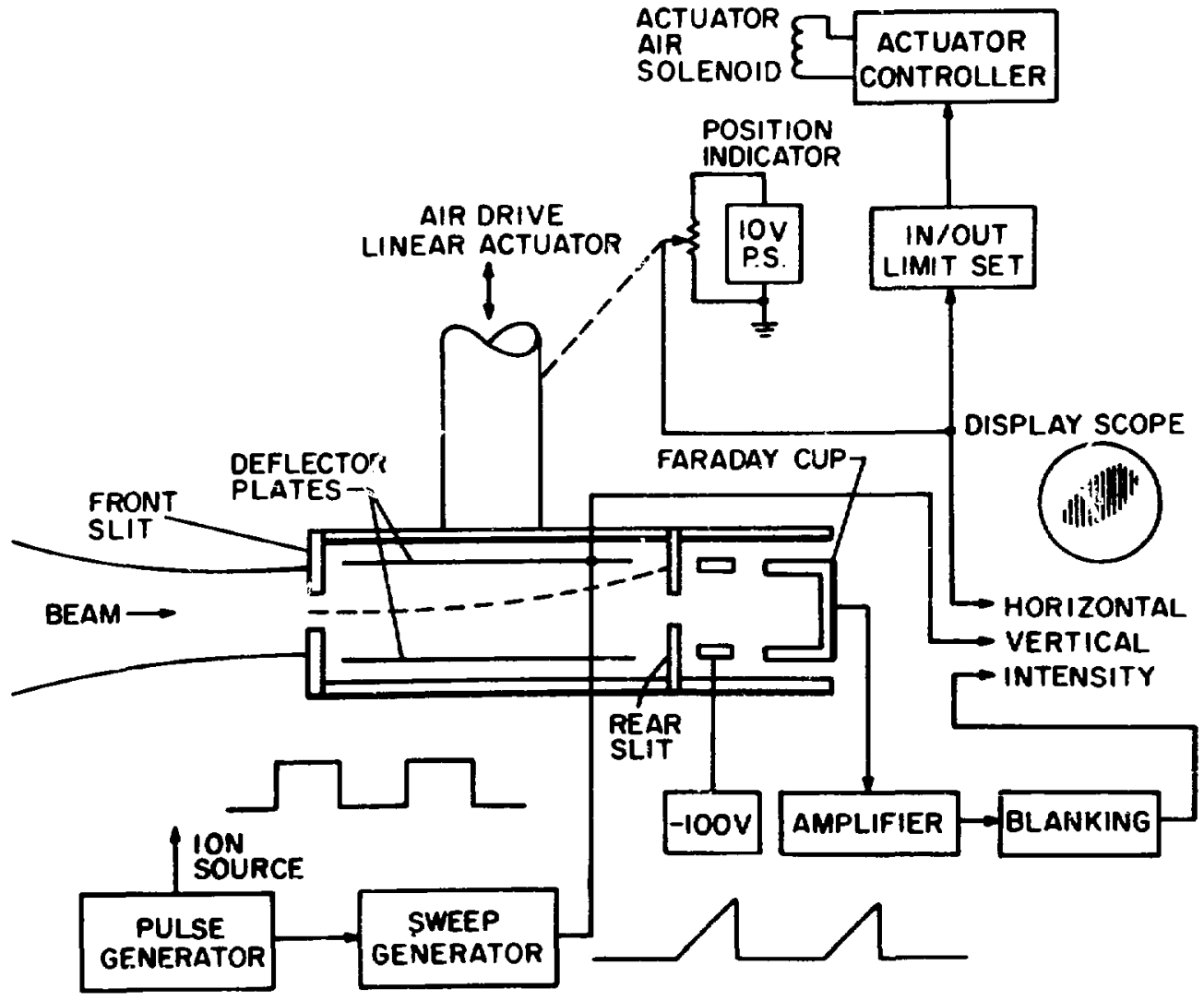

Fig. 2.

Schematic of emittance scanner. 


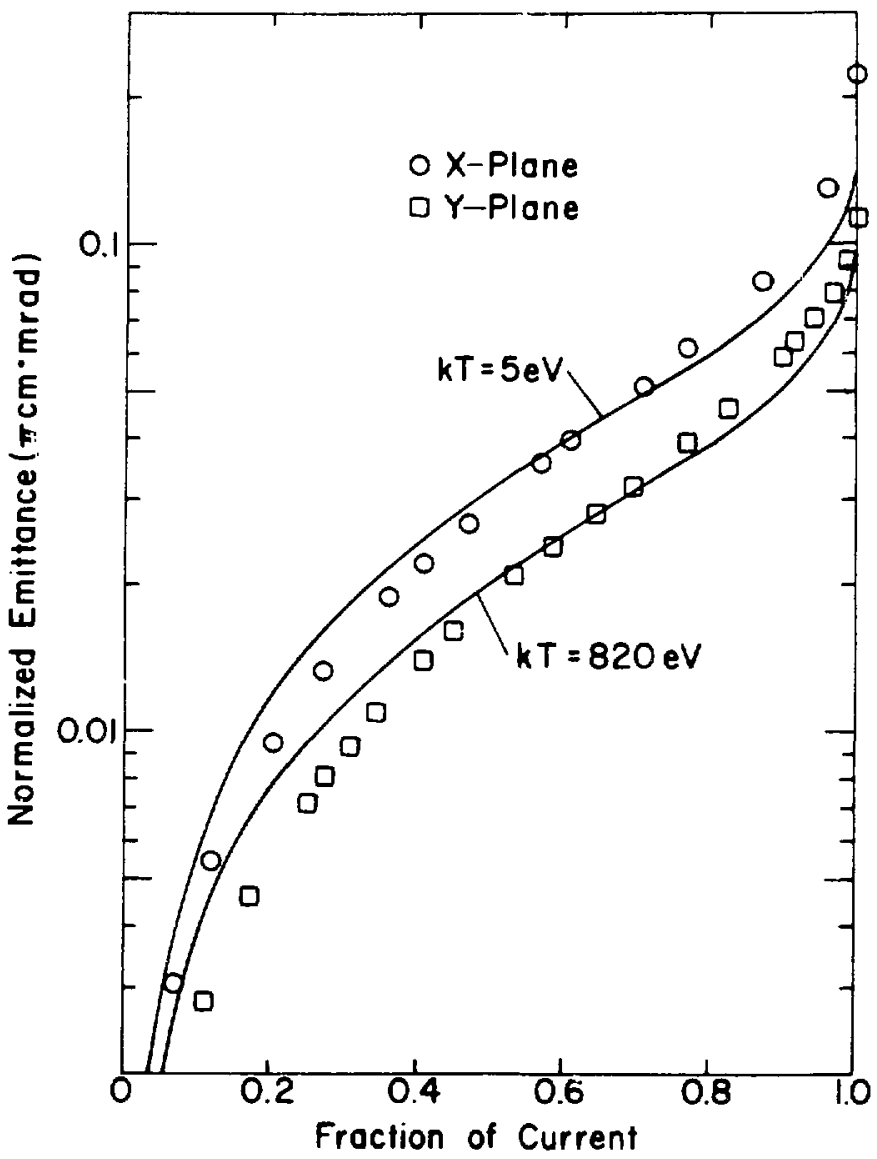

Fig. 3.

Normalized emittance vs beam fraction for a $17-\mathrm{keV}, 80-\mathrm{mA}$ beam extracted from a

$10-$ by $0.5-\mathrm{mm}^{2}$ slit. The curves are calculated using Eq. (6) with $\mathrm{kT}=5 \mathrm{eV}$ and $820 \mathrm{eV}$ in the $x$ - and $y$-planes, respectively. in the range expected. We believe that fast $\mathrm{H}^{-}$ions from the cathodes make resonant charge-exchange collisions with $H$ atoms in the discharge; therefore, their temperature should be that of the $\mathrm{H}$ atoms. The latter temperature is not known, but the Franck-Condon energy for collisional dissociation ${ }^{6}$ of $\mathrm{H}_{2}$ is $>2 \mathrm{eV} /$ atom. For the $y$-plane. $k T=820 \mathrm{eV}$; however, we know that the $x$ - and $y$-plane emittances are coupled by the bending magnet, so that the larger $x-p l$ ane emittance masks that of the $y-p l a n e$. The large derived ion temperature reflects the small initial radius of $0.025 \mathrm{~cm}$ in the $y$-plane. The ion energies at the measurement plane are about an electron volt in both planes as a consequence of the transformation between the emission and the measurement planes.

The emittance of beams ${ }^{8}$ extracted from a $1.5-m m-d i a m$ aperture fits the aperture-theory curve of $F$ ig. 1 much closer (Fig. 4). A temperature of about $5 \mathrm{eV}$ in both $\mathrm{planes}$ is derived for a 14-keV, 31-mA beam under conditions of a relatively quiet discharge voltage $( \pm 4 \%$ noise, $1-M H z$ bandwidth $)$. For a noisy discharge ( $\pm 20 \%$ noise) the emittance of a 15-kev, 25-mA beam was about three times larger, and the derived temperature was $50 \mathrm{eV}$ in both planes. 


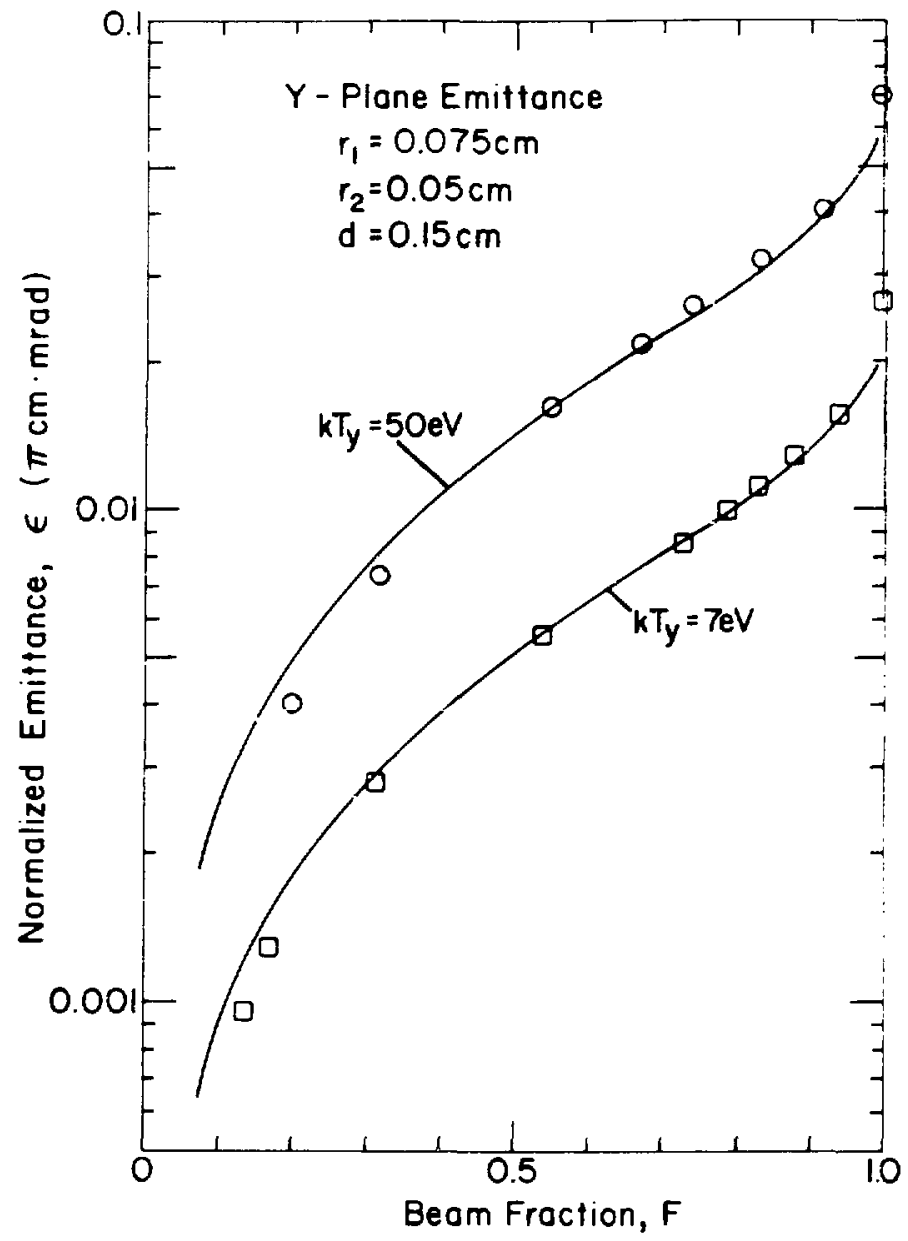

Fig. 4 .

Normalized $y$-plane emittance vs beam fraction for a noisy beam (circles) and for a quiet beam (squares) extracted from a $1.5-\mathrm{mm}$ aperture. The aperture-theory curve of $\mathrm{Fig} .1$ has been normalized to the data at $F=63 \%$, giving $k T=7 \mathrm{eV}$ (quiet beam) and $k T=50 \mathrm{eV}$ (noisy bean).

Similar results were obtained in the $x-p l a n e$.

\section{DISCUSSION}

A noisy discharge may lead to fluctuations in the emitting plasma surface, with subsequent variations in the ion crtics, and to incomplete neutralization in the transport, the combined effect being a time-averaged increase in the emittance. It is interesting that the data for both quiet and noisy discharges (Fig. 4) are consistent with the "Maxwellian" model. The emittance of the slit beam is more nearly consistent with the "Gaussian" model, but we have no evidence that the plasma density at the emitter is not uniform. Magnet aberrations coupie the $x$ - and $y-p l a n e$ emittances, causing significant phasespace distortions for slit beams because of the large initial asymmetry in emittance at the emission plane.

For aperture beams, the coupling has a smaller effect because of the symmetry in initial emittances.

Results for the various models are given in Table $I$. We have included the emittance for $63 \%$ of the beam in a single plane, $E(63 \%)$. The curves of emittance vs beam fraction for the $K-V$, the "Maxwellian," and the "Gaussian" models are presented in Fig. 5. The curve for the $K-V$ model is seen to differ significantly from the other two curves and from our measured data (Figs. 3 and 4 ). 
SUMMARY OF RESULTS FOR VARIOUS EMITTANCE MODELS

\begin{tabular}{|c|c|c|c|c|c|c|c|}
\hline Model & $\begin{array}{c}\text { Threshoid } \\
\mathrm{t}\end{array}$ & $\begin{array}{l}\text { Beam Fraction, } F(n) \\
\text { where } n=\varepsilon / \varepsilon 4 \text { rms }\end{array}$ & $\begin{array}{l}F(1) \\
(\%) \\
\end{array}$ & $\begin{array}{l}\left\langle x^{2}\right\rangle \\
\left(R^{2}\right)\end{array}$ & $\begin{array}{l}\left\langle\dot{x}^{2}\right\rangle \\
(k T / m) \\
\end{array}$ & $\begin{array}{c}\varepsilon_{4 r m s} \\
\left(R \sqrt{\left.k T / m c^{2}\right)}\right.\end{array}$ & $\begin{array}{c}E(63 \%) \\
\left(R \sqrt{k T / m c^{2}}\right) \\
\end{array}$ \\
\hline \multicolumn{8}{|l|}{ 'Maxwellian" } \\
\hline Slit & $\begin{array}{l}\exp \left(-m \dot{x}^{2} / 2 k T\right) \\
\text { for }|x| \leq R,|\dot{x}|<\infty\end{array}$ & $\operatorname{erf}(\pi n / \sqrt{6}$ & 93 & $1 / 3$ & 1 & $4 / \sqrt{3}=2.31$ & 1.14 \\
\hline Aperture & $\begin{array}{l}\sqrt{1-x^{2} / R^{2}} \exp \left(-m \dot{x}^{2} / 2 k T\right) \\
\quad \text { for }|x| \leq R,|\dot{x}|<\infty\end{array}$ & Fig. i & 89 & $1 / 4$ & 1 & 2 & 1.05 \\
\hline \multicolumn{8}{|l|}{ "Gaussian" } \\
\hline $\begin{array}{l}\text { Slit or } \\
\text { Aperture }\end{array}$ & $\begin{array}{l}\exp \left(-3 x^{2} / 2 R^{2}-m \dot{x}^{2} / 2 k T\right) \\
\text { for }|x|<\infty,|\dot{x}|<\infty\end{array}$ & $1-e^{-2 n}$ & 86 & $1 / 3$ & 1 & $4 / \sqrt{3}=2.31$ & 1.15 \\
\hline $\begin{array}{l}\text { Kapchinskij- } \\
\text { Vladmirskii }\end{array}$ & $\begin{array}{l}1 \\
\text { for }\end{array}$ & & & & & & \\
\hline & $(x / R)^{2}+m \dot{x}^{2} / 4 k T \leq 1$ & $\eta$ & 100 & $1 / 4$ & 1 & 2 & 1.26 \\
\hline
\end{tabular}




\section{VII . CONCLUSION}

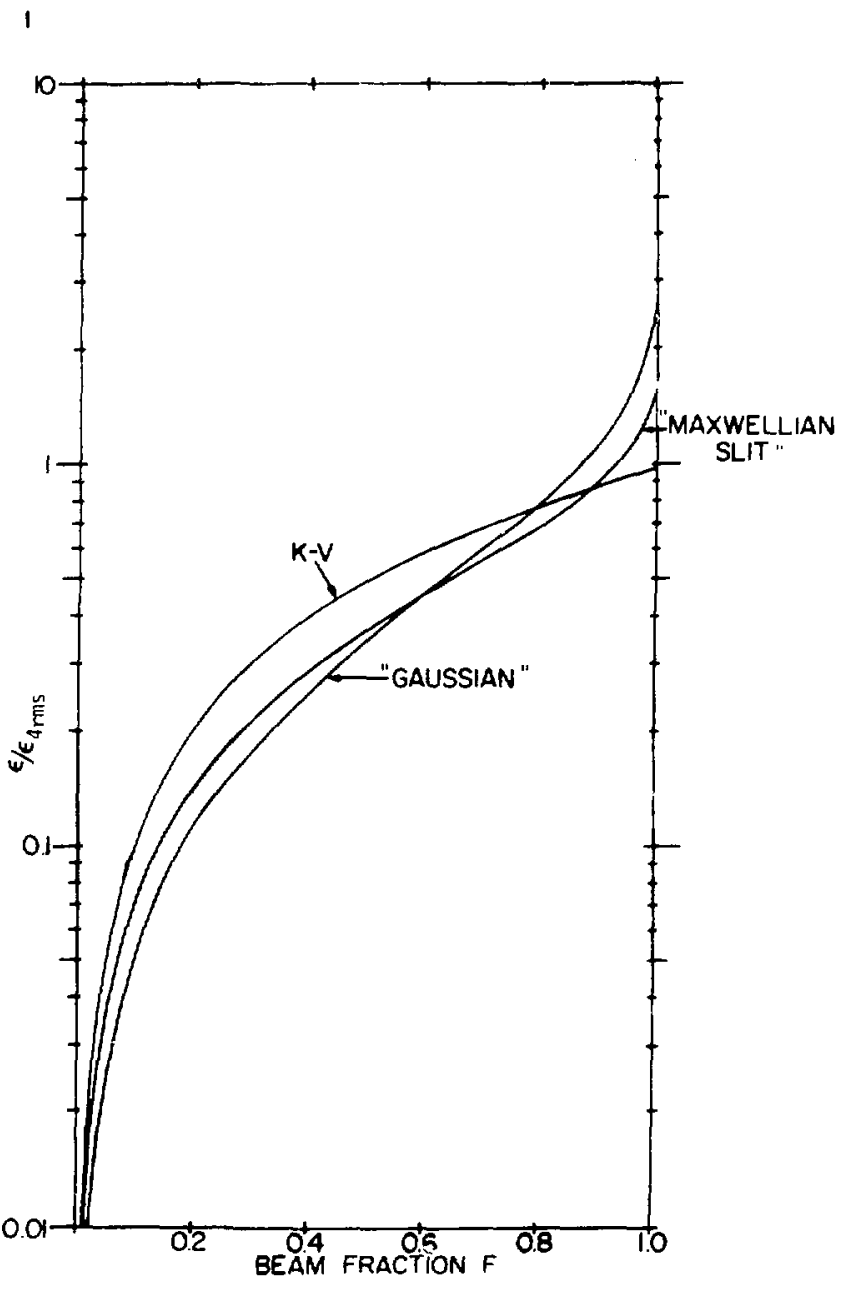

Fig. 5.

Normalized emittance vs beam fraction for several of the models discussed in the text.
The emittance as a function of beam fractinn foi our $\mathrm{H}^{-}$ion beam is reasonably described by the "Maxwellian" model of ion density at the plasma surface, even for cases where the emittance is dominated by effects of magnet aberrations or dis. charge noise. In the absence of emittance growth after extraction, the dependence of $\varepsilon$ on $F$ has been shown theoretically to be invariant in trarsport. For some conditions, the derived ion temperature (about $4 \mathrm{eV}$ ) is near that expected from considerations of ion formation mechanisms; however, no independent measurement of ion temperature has been made. For other conditions, the derived ion temperature is greater than can rea-

sonably be expected in the plasma. The fit of the theory still can be good, indicating that the distribution is equivalent to that for the "Maxwellian" model. To the extent that the theory fits the data, the emittance can be given by a single number, for example, $\varepsilon_{4 r m s}$. The beam fraction at a given emittance then is given by theory, just as the temperature describes the complete distribution of particle energies in a gas at thermal equilibrium. 


\section{REFERENCES}

1 P. W. Allison, "Experiments with a Dudnikov-Type $H^{-}$I on Source," Proc. Jymp. on the Production and Neutralization of Negative Hydrogen Ions and Beams, Upton, New York, September 26-30, 1977 (Brookhaven National Laboratory, Upton, New York, 1977), BNL-50727, p. 119.

2. J. D. Lawson, The Physics of Charged Particle Beams, (Oxford Press, 0.ford, 1977), pp. 175-176.

3. I. M. Kapchinskii and V. V. Vladimirskii, "Limitations of Proton Beam Current in a Strong Focusing Linear Accelerator Associated with the Beam Space Charge," Proc. Int. Conf. on High Energy Accelerators and Instrumentation, CERN, Geneva, 1959, p. 274.

4. Pierre Lapostolle, "Possible Emittance Increase through Filamentation due to Space Charge in Continuous Beams", 1971 Particle Accelerator Conf., Chicago, Illinois, March 1-3, 1971, IEEE Trans. Nuci. Sci. 18, 1101 (1971).

5. F. W. Allison, H. V. Smith, Jr., and J. D. Sherman, "H" Inn Source Research at Los Alamcs," Proc. 2nd Int. Symp. on the Production and Neutralization of Negative Hydrogen I ons and Beams, Upton, New York, October 6-10, 1980 (Brookhaven National Laboratory, Upton, New York, 1981) BNL-51304, p. 171 .

6. H. S. W. Massey, "Electronic and Ionic Impact Phenomena, Vol. II", (0xford Press, Oxford, 1969), p. 883 .

7. J. D. Sherman and P. W. Allison, "A Study of $90^{\circ}$ Bending Miagnet for $H^{-}$Beams," 1979 Particle Accelerator Conf., San Franciscc, Cal ifornia, March 12-13, 1979, IEEE Trans. Nucl. Sci. 26, 3916 (1979).

8. J. D. Sherman, P. W. Allison and H. V. Cm,th, Jr., "H" Beam Formation from a Penning Discharge Surface Plasma Source using Circular EmissionExtractor Electrodes," Proc. 2nd Int. Symp. on the Production and Neutralization of Negative Hydrogen Ions and Beams, Upton, New York, October 6-10, 1980 (Brookhaven National Laboratory, Upton, New York, 1981), BNL-51304, p.18c 